\title{
Spectral and Scattering Theory of Schrödinger Operators Related to the Stark Effect
}

\author{
J. E. Avron^ and I. W. Herbst ${ }^{\star \star}$ \\ Joseph Henry Laboratories of Physics, Princeton University, Princeton, New Jersey 08540, USA
}

\begin{abstract}
We analyze the spectral properties and discuss the scattering theory of operators of the form $H=H_{0}+V, H_{0}=-\Delta+\boldsymbol{E} \cdot \boldsymbol{x}$. Among our results are the existence of wave operators, $\Omega_{ \pm}\left(H, H_{0}\right)$, the nonexistence of bound states, and a (speculative) description of resonances for certain classes of potentials.
\end{abstract}

\section{Introduction}

Since 1951, when Kato [10] initiated the mathematical analysis of Schrödinger operators of the form $-\Delta+V(\boldsymbol{x})$ [with $V(\boldsymbol{x}) \rightarrow 0$ as $|\boldsymbol{x}| \rightarrow \infty$ in some sense] this class of operators has become quite well understood (see $[15,17,18]$ for references to original contributions). It is the purpose of this paper to initiate a study of the class of operators which result from the above by the addition of a potential corresponding to a constant electric field. We call such operators Stark Hamiltonians. They are of the form

$$
H=H_{0}+V, \quad H_{0}=-\Delta+\boldsymbol{E} \cdot \boldsymbol{x} .
$$

Assuming a point nucleus, the hydrogen Stark effect $[6,13]$ is described by the Coulomb potential $V(\boldsymbol{x})=-Z /|\boldsymbol{x}|$.

We begin our analysis in Section II with a discussion of the operator $H_{0}$. In most quantum mechanics books the potential $\boldsymbol{E} \cdot \boldsymbol{x}$ is treated as a perturbation of $-\Delta+V$. However, this perturbation is not small in any obvious mathematical sense (no matter the size of $\boldsymbol{E}$ ). (See, however, [18] and [25] for the theory of spectral concentration.) Thus we find it useful to treat $V$ as a perturbation of $H_{0}$. It will become clear in later sections that for the classes of potentials which we consider, many of the properties of $H_{0}$ remain invariant under the addition of $V$.

In Section III we consider a class of potentials for which the wave operators $\Omega_{ \pm}\left(H, H_{0}\right)$ exist. In contrast to the situation where $\boldsymbol{E}=0$, the Coulomb potential is included in this class. In fact a large class of potentials which in certain directions approach infinity as $|\boldsymbol{x}| \rightarrow \infty$ (without oscillation) is allowed.

* Wigner Fund Fellow, Technische University of Berlin

$\star \star$ Supported in part by NSF Grant MPS 74-22844 
In Section IV we give criteria for $V$ to be compact relative to $H_{0}$. We study a subclass of such $V$ 's which are analytic (in a strip) in the variable parallel to $\boldsymbol{E}$. These potentials turn out to be especially nice. We analyze them by a method motivated by the Balslev-Combes [4] treatment of dilatation-analytic perturbations of the Laplacian. We speculate on a mathematical description of resonances provided by this technique. While the Coulomb potential does not fall into this class, the potential produced by a nucleus of finite size with, for example, a gaussian charge distribution does. Among other results our analysis shows the absence of singular continuous spectrum for these potentials.

In Section $\mathrm{V}$ we consider a large class of potentials characterized most importantly by the condition that

$$
|\boldsymbol{E}|-\left(\nabla_{\|} V\right)(\boldsymbol{x}) \geqq \delta>0
$$

for $x_{\|}$large enough. (Here $\|$stands for the direction parallel to $E$.) We show that for such potentials (see Section $\mathrm{V}$ for an exact description), $H$ has no eigenstates. This generalizes a result of Titchmarsh [23] who proved the absence of bound states for the hydrogen Stark effect. His analysis makes explicit and crucial use of the separability of the problem in parabolic coordinates. Agmon [1] has also announced the non-existence of point spectrum for a general class of potentials. The proof of the relevant theorem has however not appeared (but see [2] where similar results are proved).

\section{The Operator $H_{0}=-\Delta+\boldsymbol{E} \cdot \boldsymbol{x}$}

In this section we set $\boldsymbol{E}=\varepsilon_{0} \boldsymbol{e}_{x}$ where $\boldsymbol{e}_{x}$ is a unit vector in the $x$ direction. We write $\boldsymbol{x}=\left(x, \boldsymbol{x}_{\perp}\right)$ and $-i \boldsymbol{\nabla}=\left(p, \boldsymbol{p}_{\perp}\right)$. Thus, we have $H_{0}=p^{2}+\boldsymbol{p}_{\perp}^{2}+\varepsilon_{0} x$. We assume for convenience that $\varepsilon_{0}>0$.

Let $H_{0}^{\sim}$ be the self-adjoint operation of multiplication by $q_{1}^{2}+q_{2}^{2}+\varepsilon_{0} x$ on $L^{2}\left(\mathbb{R}^{3}, d^{2} q d x\right)$ and let $U_{\|} \equiv e^{i p^{3} / 3 \varepsilon_{0}}$ and $\mathscr{F}_{\perp}$ be the unitary operation of (partial) Fourier transformation with respect to the variables perpendicular to $\boldsymbol{E}$. Define $U=\mathscr{F}_{\perp} U_{\|}$. We then have

Theorem 1.1. a) $\left.(-\Delta+\boldsymbol{E} \cdot \boldsymbol{x})\right|_{C_{0}^{\infty}\left(\mathbb{R}^{3}\right)}$ is essentially self-adjoint. We denote its closure by $H_{0}$.

b) $H_{0}$ is unitarily equivalent to $H_{0} \tilde{\text {. Explicitly }}$

$H_{0}=U H_{0} U^{-1}$.

c) The spectrum of $H_{0}$ is purely absolutely continuous with $\sigma\left(H_{0}\right)=(-\infty, \infty)$.

Proof. Let $\mathscr{S}=\mathscr{S}\left(\mathbb{R}^{3}\right)$ be the Schwartz space of rapidly decreasing $C^{\infty}$ functions and define

$$
\begin{aligned}
& A=\left(-\Delta+\varepsilon_{0} x\right) \uparrow_{\mathscr{S}} \\
& B=H_{0} \uparrow_{\mathscr{S}} .
\end{aligned}
$$

Note that $U$ maps $\mathscr{S}$ onto $\mathscr{S}$. By explicit calculation we have

$$
A=U B U^{-1} \text {. }
$$


Since $\mathrm{H}_{0}{ }^{\sim}$ is a self-adjoint extension of $B, U H_{0}{ }^{\sim} U^{-1}$ is a self-adjoint extension of $A$. If $C$ is another self-adjoint extension of $A$ then $U^{-1} C U$ is a self-adjoint extension of $B$. Since $\mathscr{S}$ is clearly a core for $H_{0}$ 作 have $U^{-1} C U=H_{0}$ or $C=U H_{0} U^{-1}$. Thus $A$ has one and only one self-adjoint extension, namely $U H_{0} U^{-1}$. The proof of a) and b) thus reduces to showing that $\left(A \uparrow_{C_{0}^{\infty}\left(\mathbb{R}^{3}\right)}\right)^{-}=\bar{A}$ which is trivial. The proof of c) follows trivially from b) and the explicit form of $H_{0}$.

We remark that the continuum "eigenfunctions" of $-\Delta+\varepsilon_{0} x$ are easily calculated using the explicit form of $U_{\|}$. We have

$$
\left(-\Delta+\varepsilon_{0} x\right) \psi_{\boldsymbol{q}_{0}, x_{0}}(\boldsymbol{x})=\left(\boldsymbol{q}_{0}^{2}+\varepsilon_{0} x_{0}\right) \psi_{\boldsymbol{q}_{0}, x_{0}}(\boldsymbol{x})
$$

where

$$
\psi_{\boldsymbol{q}_{0}, x_{0}}(\boldsymbol{x})=e^{i \boldsymbol{q}_{0} \cdot \boldsymbol{x}_{\perp}} \varepsilon_{0}^{1 / 3} A\left(\varepsilon_{0}^{1 / 3}\left(x-x_{0}\right)\right)
$$

and $A(x)$ is the Airy function

$$
A(x)=\lim _{N \rightarrow \infty}(2 \pi)^{-1} \int_{-N}^{N} \exp i\left\{\left(t^{3} / 3\right)+x t\right\} d t .
$$

It will be useful in Section III to have a simple expression for the propagator $e^{-i t H_{0}}$, which we now derive. We write

$$
\begin{aligned}
e^{-i t H_{0}} & =e^{i p^{3} / 3 \varepsilon_{0}} e^{-i t\left(p_{\perp}^{2}+\varepsilon_{0} x\right)} e^{-i p^{3} / 3 \varepsilon_{0}} \\
& =e^{-i t \varepsilon_{0} x} e^{i\left(p-\varepsilon_{0} t\right)^{3} / 3 \varepsilon_{0}} e^{-i t p^{2}} e^{-i p^{3} / 3 \varepsilon_{0}} \\
& =e^{-i t \varepsilon_{0} x} e^{-i t\left(p^{2}+p_{\perp}^{2}\right)} e^{i \varepsilon_{0} t^{2} p} e^{-i \varepsilon_{0}^{2} t^{3} / 3}
\end{aligned}
$$

This has a simple interpretation in terms of the Heisenberg operators $\boldsymbol{x}(t)=$ $e^{i t H_{0}} x e^{-i t H_{0}}$ and $p(t)=e^{i t H_{0}} p e^{-i t H_{0}}$ which can be calculated from (2.4):

$$
\begin{aligned}
& \boldsymbol{p}(t)=\boldsymbol{p}-\boldsymbol{E} t \\
& \boldsymbol{x}(t)=\boldsymbol{x}+2 \boldsymbol{p} t-\boldsymbol{E} t^{2} .
\end{aligned}
$$

These are of course the familiar solutions to Newton's equations

$$
d \boldsymbol{p}(t) / d t=-\boldsymbol{E}, \quad d \boldsymbol{x}(t) / d t=2 \boldsymbol{p}(t) .
$$

Note that Equation (2.5) in turn determines $e^{i t H_{0}}$ up to a multiplicative phase factor.

\section{Scattering: The Existence of Wave Operators}

In this section we consider a class of potentials for which a "reasonable" scattering theory can be developed. To emphasize the physical interpretation we also discuss the classical situation.

We are interested in the existence of the strong limit of $e^{i t H} e^{-i t H_{0}}$ as $t \rightarrow \pm \infty$, or what is the same thing, in the existence (for every asymptotic state $f \in L^{2}$ ) of a state $\psi$ such that $\left\|e^{-i t H} \psi-e^{-i t H_{0}} f\right\| \rightarrow 0$. Given a point $Q=(\boldsymbol{x}, \boldsymbol{p})$ in $\mathbb{R}^{6}$, let $\left(x_{Q}^{0}(t), p_{Q}^{0}(t)\right)$ be the solution to the free Equation (2.6) with initial data $Q$. In analogy to the quantum mechanical case, in classical mechanics we are interested in the 
existence of an interacting orbit $\left(\boldsymbol{x}_{Q}(t), \boldsymbol{p}_{Q}(t)\right)$ for each asymptotic "state" $Q$ so that the difference between $\left(\boldsymbol{x}_{Q}(t), \boldsymbol{p}_{Q}(t)\right)$ and $\left(\boldsymbol{x}_{Q}^{0}(t), \boldsymbol{p}_{Q}^{0}(t)\right)$ tends to zero as $t \rightarrow \infty$. In this regard we have the following theorem.

Theorem 3.1. Let $\boldsymbol{E}=\varepsilon_{0} \boldsymbol{e}_{x}$ with $\varepsilon_{0}>0$. Suppose $\boldsymbol{F}: \mathbb{R}^{3} \rightarrow \mathbb{R}^{3}$ is continuously differentiable and satisfies

a) $|\boldsymbol{F}(\boldsymbol{x})| \leqq c\left(1+\left|\boldsymbol{x}_{\perp}\right|\right)^{n}(1+|x|)^{-(n / 2)-1-\varepsilon} \quad x \leqq 0$;

b) $\left|\partial_{j} F_{k}(x)\right| \leqq c^{\prime}\left(1+\left|\boldsymbol{x}_{\perp}\right|\right)^{m}(1+|x|)^{-(m / 2)-1-\delta} \quad x \leqq 0$

where $m, n \geqq 0, \varepsilon, \delta>0$. Then for each $Q=(\boldsymbol{x}, \boldsymbol{p})$ in $\mathbb{R}^{6}$ there exists a unique solution $(\boldsymbol{x}(t), \boldsymbol{p}(t))=\left(\boldsymbol{x}_{Q}(t), \boldsymbol{p}_{Q}(t)\right)$ of the equations

$$
d \boldsymbol{p}(t) / d t=-\boldsymbol{E}+\boldsymbol{F}(\boldsymbol{x}(t)), \quad d \boldsymbol{x}(t) / d t=2 \boldsymbol{p}(t)
$$

for large time, satisfying

$$
\lim _{t \rightarrow+\infty}\left|\boldsymbol{x}_{Q}^{0}(t)-\boldsymbol{x}_{Q}(t)\right|+\left|\boldsymbol{p}_{Q}^{0}(t)-\boldsymbol{p}_{Q}(t)\right|=0 .
$$

Proof. Our proof follows $[8,22]$. Consider the function

$$
f(t, y)=F\left(y+x+2 p t-\varepsilon_{0} e_{x} t^{2}\right)
$$

and the space $S_{T}=\left\{y(\cdot): y(\cdot)\right.$ is a continuous map from $(T, \infty)$ into $\mathbb{R}^{3}$ satisfying $\left.\|\boldsymbol{y}(\cdot)\|_{\infty, T}=\sup _{t>T}|\boldsymbol{y}(t)| \leqq 1\right\}$. We will choose $T>0$ so that

$$
\boldsymbol{A}(\boldsymbol{y}(\cdot))(t)=\int_{t}^{\infty} d s \int_{s}^{\infty} d u f(u, y(u))
$$

is a strict contraction from $S_{T}$ into $S_{T}$. Choose $T$ large enough so that $(x+2 p t-$ $\left.\varepsilon_{0} e_{x} t^{2}\right) \cdot e_{x}<-1$, all $t \geqq T$. From a) we have

$$
\|\boldsymbol{A}(\boldsymbol{y}(\cdot))\|_{\infty, T} \leqq \int_{T}^{\infty} d s \int_{s}^{\infty} d u|\boldsymbol{f}(u, \boldsymbol{y}(u))|<c_{1} T^{-2 \varepsilon}
$$

and from $b$ )

$$
\left\|A\left(y_{1}(\cdot)\right)-A\left(y_{2}(\cdot)\right)\right\|_{\infty, T} \leqq c_{2} T^{-2 \delta}\left\|y_{1}(\cdot)-y_{2}(\cdot)\right\|_{\infty, T} .
$$

By the contraction mapping fixed point theorem there is a unique solution $\boldsymbol{y}_{Q}(\cdot)$ in $S_{T}$ (if $T$ is large enough) to the equation

$$
A(y(\cdot))=y(\cdot) \text {. }
$$

Defining

$$
\boldsymbol{x}_{Q}(t)=\boldsymbol{y}_{Q}(t)+\boldsymbol{x}_{Q}^{0}(t), \quad \boldsymbol{p}_{Q}(t)=2^{-1} d \boldsymbol{x}_{Q} / d t
$$

(3.1) and (3.2) easily follow. It is also easy to see that any solution of (3.1) satisfying (3.2) also yields a $\boldsymbol{y}(\cdot)$ satisfying (3.5). A local uniqueness theorem then shows that $\boldsymbol{y}(t)=\boldsymbol{y}_{Q}(t)$ for $t>T$. This completes the proof.

Remarks. a) The fact that the forces are allowed to grow in some directions (and are essentially arbitrary for $x>0$ ) means that in general global solutions to (3.1) will not exist for arbitrary initial conditions. In quantum mechanics this should have as a consequence that $(-\Delta+\boldsymbol{E} \cdot \boldsymbol{x}+V(\boldsymbol{x})) \uparrow_{C_{0}^{\infty}\left(\mathbb{R}^{3}\right)}$ is not in general essentially self-adjoint [17]. 
b) Clearly one can arrange $\boldsymbol{F}(\boldsymbol{x})$ to satisfy the conditions a) and b) of the theorem and still have unbounded orbits which are not asymptotic to any free orbit. In quantum mechanics one should then expect that the wave operators $\Omega_{ \pm}\left(H, H_{0}\right)$ are not complete.

We now go on to the quantum case.

Theorem 3.2. Suppose $V$ is a real measurable function on $\mathbb{R}^{3}$ satisfying

a) $\left(1+x^{2}\right)^{-N} V \in L^{2}\left(\mathbb{R}^{3}\right)$ for some $N>0$.

b) For every $c>0$ there exists a $t_{0}>0$ with

$$
\int_{t_{0}}^{\infty} d t t^{-3 / 2}\left(\int_{|x| \leqq c t} d^{3} x\left|V\left(x-\varepsilon_{0} t^{2}, \boldsymbol{x}_{\perp}\right)\right|^{2}\right)^{1 / 2}<\infty .
$$

Let $H$ be any self-adjoint extension of

$$
\left(H_{0}+V(\boldsymbol{x})\right) \uparrow_{C_{0}^{\infty}\left(\mathbb{R}^{3}\right)}, \quad H_{0}=-\Delta+\varepsilon_{0} x, \quad \varepsilon_{0}>0 .
$$

Then the wave operators $\Omega_{ \pm}\left(H, H_{0}\right)=\underset{t \rightarrow \pm \infty}{s-\lim _{t \rightarrow \infty}} e^{i t H} e^{-i t H_{0}}$ exist.

Corollary 3.3. Under the same conditions as Theorem 3.2, $\sigma_{\text {a.c. }}(H)=(-\infty, \infty)$.

Before proving Theorem 3.2 we note the following sufficient condition for the existence of $\Omega_{ \pm}$(compare with Theorem 3.1).

Lemma 3.4. Suppose $V=V_{1}+V_{2}$ where
a) $V_{1} \in L^{2}$
b) $\left(1+x^{2}\right)^{-N} V_{2} \in L^{2}$ for some $N$ and for $x \leqq 0$
$\left|V_{2}(\boldsymbol{x})\right| \leqq c\left(1+\left|\boldsymbol{x}_{\perp}\right|\right)^{n}(1+|x|)^{-1 / 2(n+1+\varepsilon)}$ for some $n \geqq 0, \varepsilon>0$.

Then $V$ satisfies the requirements of Theorem 3.2 and $\Omega_{ \pm}\left(H, H_{0}\right)$ exist.

Proof of Theorem 3.2. It is enough to prove convergence of $\Omega(t)=e^{i t H} e^{-i t H_{0}}$ on the dense set $\mathscr{D}=\left\{\psi: \hat{\psi} \in C_{0}^{\infty}\left(\mathbb{R}^{3}\right)\right\}$. By standard arguments one need only prove convergence of the integral $\int_{t_{0}}^{\infty}\left\|\frac{d}{d t} \Omega(t) \psi\right\| d t$ for some $t_{0}>0$. Using the fact that $e^{-i t H_{0}}: \mathscr{S}\left(\mathbb{R}^{3}\right) \rightarrow \mathscr{S}\left(\mathbb{R}^{3}\right)$ and the easily proved fact that $H \uparrow_{\mathscr{S}}=\left(H_{0}+V\right) \uparrow_{\mathscr{S}}$, we can differentiate $e^{i t H}$ so that we need only prove integrability of

$$
f_{\psi}(t) \equiv\left\|V(\boldsymbol{x}) e^{-i t H_{0}} \psi\right\|
$$

for $t \geqq t_{0}$. From Equation (2.4) we have

$$
f_{\psi}(t)=\left\|V\left(x-\varepsilon_{0} t^{2}, \boldsymbol{x}_{\perp}\right) \psi_{t}\right\|
$$

where $\psi_{t}=e^{i t \Delta} \psi$. The behavior of $\psi_{t}(\boldsymbol{x})$ for large $t$ is well known $[5,19]$. From the explicit form of the kernel of $e^{i t \Delta}$ one has $\left\|\psi_{t}\right\|_{\infty} \leqq c t^{-3 / 2}$. We give a short proof of the fact that for $|\boldsymbol{x}| \geqq c t$ (where $c$ depends on $\psi$ )

$$
\left|\psi_{t}(\boldsymbol{x})\right| \leqq D_{N}\left(1+\boldsymbol{x}^{2}+t^{2}\right)^{-N}
$$


Suppose supp $\hat{\psi} \subseteq\{\boldsymbol{p}:|\boldsymbol{p}|<R\}$. We have then for $|\boldsymbol{x}|>2 R t$

$$
\begin{aligned}
\psi_{t}(\boldsymbol{x}) & =(2 \pi)^{-3 / 2} \int e^{-i\left(\boldsymbol{p}^{2} t-\boldsymbol{p} \cdot \boldsymbol{x}\right)} \hat{\psi}(\boldsymbol{p}) d^{3} p \\
& =(2 \pi)^{-3 / 2} i^{N} \int e^{-i\left(\boldsymbol{p}^{2} t-\boldsymbol{p} \cdot \boldsymbol{x}\right)}\left[(\partial / \partial k)(|\boldsymbol{x}|-2 k t)^{-1}\right]^{N} \hat{\psi}(\boldsymbol{p}) d^{3} p
\end{aligned}
$$

where $k=\boldsymbol{p} \cdot \boldsymbol{x} /|\boldsymbol{x}|$. Thus

$$
\left|\psi_{t}(\boldsymbol{x})\right| \leqq c_{N} \sum_{i=0}^{N} t^{i}(|\boldsymbol{x}|-2 R t)^{-N-i} \int\left|\hat{\psi}^{(N-i)}(\boldsymbol{p})\right| d^{3} p
$$

where $\hat{\psi}^{(j)}=\left(\boldsymbol{x}|\boldsymbol{x}|^{-1} \cdot \boldsymbol{\nabla}\right)^{j} \hat{\psi}$. Thus if $|\boldsymbol{x}|>(2 R+\varepsilon) t$, (3.11) gives

$$
\left|\psi_{t}(\boldsymbol{x})\right| \leqq c_{N}^{\prime}(\varepsilon)(|\boldsymbol{x}|+t)^{-N}
$$

and thus (3.9) follows.

To show that $f_{\psi}$ is integrable on $\left[t_{0}, \infty\right)$ we use (3.9) to show that

$$
\left\|V\left(x-\varepsilon_{0} t^{2}, \boldsymbol{x}_{\perp}\right)\left(1-\chi_{c t}\right) \psi_{t}\right\| \leqq c^{\prime}\left\|V\left(1+\boldsymbol{x}^{2}\right)^{-N}\right\|_{2} t^{-M}
$$

for $c>2 R, \chi_{c t}(x)=1$ if $|x| \leqq c t$, and zero otherwise. While

$$
\left\|V\left(x-\varepsilon_{0} t^{2}, x_{\perp}\right) \chi_{c t} \psi_{t}\right\| \leqq c^{\prime} t^{-3 / 2}\left\|V\left(x-\varepsilon_{0} t^{2}, x_{\perp}\right) \chi_{c t}\right\|
$$

which yields exactly the integral (3.6).

\section{Relatively Compact and Analytic Potentials}

We begin this section with a discussion of relatively compact perturbation of $-\Delta+G(x)$ where $G$ is a quite general multiplication operator.

Definition 4.1. $L_{0}^{q}\left(\mathbb{R}^{3}\right)$ is the set of all $L^{q}\left(\mathbb{R}^{3}\right)$ functions with compact support. $L^{q}+L^{\infty}$ is the set of all functions $f$ such that $f=f_{1}+f_{2}$ with $f_{1} \in L^{q}$ and $f_{2} \in L^{\infty}$. $L_{0, c}^{q}\left(\mathbb{R}^{3}\right)$ is the set of all $f$ such that for each $\varepsilon>0, f=f_{1 \varepsilon}+f_{2 \varepsilon}$ with $f_{1 \varepsilon} \in L_{0}^{q},\left\|f_{2 \varepsilon}\right\|_{\infty} \leqq \varepsilon$.

Theorem 4.2. Suppose $G$ and $V$ are real multiplication operators such that

a) for some $\alpha>0 \mathrm{Ge}^{-\alpha|x|} \in L^{p}\left(\mathbb{R}^{3}\right)$

b) $V \in L_{0, c}^{q}$

where either $p^{-1}+q^{-1}=2 / 3$ and $2<p, q$ or $p=2$ and $q>6$ or $q=2$ and $p>6$. Then if $H_{0}$ is some self-adjoint extension of $(-\Delta+G) \uparrow_{C_{0}^{\infty}\left(\mathbb{R}^{3}\right)}, V\left(H_{0}+i\right)^{-1}$ is compact.

Corollary 4.3. Let $H_{0}$ and $V$ be as in Theorem 4.2. Then
a) $H=\left(H_{0}+V\right) \uparrow_{\mathscr{D}\left(H_{0}\right)}$ is self-adjoint;

b) $\sigma_{\text {essential }}(H)=\sigma_{\text {essential }}\left(H_{0}\right)$.

Given Theorem 4.2, the corollary follows from standard results [11].

Proof of Theorem 4.2. Our proof is a bit subtle because the domain of $H_{0}$ is not specified. Let $\lambda=2(\alpha+1)^{2}$ and define the operator $K_{h}$ for $h \in L_{0}^{q}, e^{-\alpha|x|} G \in L^{p}$ by giving its kernel:

$$
K_{h}(x, y)=h(x)(4 \pi|x-y|)^{-1} \exp \left\{-(1+i)(\lambda / 2)^{1 / 2}|x-y|\right\} G(y) .
$$


Note that the middle term is just the kernel of $(-\Delta+i \lambda)^{-1}$. The operator $K_{h}$ is Hilbert-Schmidt. Explicitly

$$
\left\|K_{h}\right\|_{2}^{2} \leqq c \int\left|h(x) e^{\alpha|x|}\right|^{2}|x-y|^{-2} e^{-2|x-y|}\left|G(y) e^{-\alpha|y|}\right|^{2} d^{3} x d^{3} y \leqq c_{1}\left\|h e^{\alpha|x|}\right\|_{q}^{2}
$$

by Sobolev's inequality if $p^{-1}+q^{-1}=2 / 3, p, q>2$ and by Hölder's inequality if $q=2, p>6$ or $p=2, q>6$. We will show that for $V \in L_{0}^{q}$, the operator $V\left(H_{0}+i \lambda\right)^{-1}$ is Hilbert-Schmidt. This is enough to prove the theorem. To accomplish this we first show that for $h \in L_{0}^{\infty}\left(\mathbb{R}^{3}\right)$

$$
h\left(H_{0}+i \lambda\right)^{-1}=h(-\Delta+i \lambda)^{-1}-K_{h}\left(H_{0}+i \lambda\right)^{-1} .
$$

It is easily shown that $H_{0} \uparrow \mathscr{D}(-\Delta) \cap \mathscr{D}(G)=-\Delta+G$. We will use this fact in what follows. Suppose $\psi \in L_{0}^{\infty}$. Then since $(-\Delta-i \lambda)^{-1} \psi \in \mathscr{D}(G) \cap \mathscr{D}(-\Delta)$ [as a glance at the kernel of $(-\Delta-i \lambda)^{-1}$ shows] we have

$$
\begin{aligned}
\left(H_{0}-i \lambda\right)^{-1} \psi & =\left(H_{0}-i \lambda\right)^{-1}(-\Delta-i \lambda)(-\Delta-i \lambda)^{-1} \psi \\
& =\left(H_{0}-i \lambda\right)^{-1}(-\Delta+G-i \lambda-G)(-\Delta-i \lambda)^{-1} \psi \\
& =(-\Delta-i \lambda)^{-1} \psi-\left(H_{0}-i \lambda\right)^{-1} G(-\Delta-i \lambda)^{-1} \psi .
\end{aligned}
$$

Thus if $\phi \in L^{2} \cap L^{\infty}$

$$
\left(H_{0}-i \lambda\right)^{-1} h \phi=(-\Delta-i \lambda)^{-1} h \phi-\left(H_{0}-i \lambda\right)^{-1} K_{h}^{*} \phi .
$$

Since all the relevant operators appearing in Equation (4.5) are bounded, (4.5) extends to all $\phi \in L^{2}$. The adjoint of the corresponding operator equation is (4.3). We now proceed by a limiting process. Let $h_{N}(\boldsymbol{x})=V(\boldsymbol{x})$ if $|V(\boldsymbol{x})| \leqq N$ and zero otherwise. Note $h_{N} \in L_{0}^{\infty}$ so that (4.3) holds. Then since $h_{n}(-\dot{\Delta}+i \lambda)^{-1} \phi \rightarrow V(-\Delta+$ $i \lambda)^{-1} \phi$ and $K_{h_{n}}\left(H_{0}+i \lambda\right)^{-1} \phi \rightarrow K_{V}\left(H_{0}+i \lambda\right)^{-1} \phi$ we see that $\left(H_{0}+i \lambda\right)^{-1} \phi \in \mathscr{D}(V)$ and (4.3) holds with $h$ replaced by $V$. This completes the proof because Equation (4.3) displays $V\left(H_{0}+i \lambda\right)^{-1}$ as a sum of two Hilbert-Schmidt operators.

We now return to our Stark Hamiltonians and consider a class of potentials for which an analysis of the spectrum is particularly easy. Our methods are motivated by the work of Balslev and Combes [4] on dilatation-analytic perturbations of $-\Delta$. This class of perturbations is inappropriate for the study of Stark Hamiltonians so we introduce the following:

Definition 4.4. Suppose $V$ is a multiplication operator such that for almost every $\boldsymbol{x}_{\perp}, V\left(z, \boldsymbol{x}_{\perp}\right)$ is analytic in a strip $|\operatorname{Imz}|<\beta$ with $\beta>0$ and independent of $\boldsymbol{x}_{\perp}$. Let $H_{0}=-\Delta+\varepsilon_{0} x . V$ is said to be $H_{0}$-translation analytic if [with $V_{z}(\boldsymbol{x})=V\left(x+z, \boldsymbol{x}_{\perp}\right)$ ] $V_{z}\left(H_{0}+i\right)^{-1}$ is a compact analytic-operator valued function of $z$ in the strip.

Lemma 4.5. Suppose $V\left(z, \boldsymbol{x}_{\perp}\right)$ is analytic in the strip $|\operatorname{Im} z|<\beta$ for almost every $\boldsymbol{x}_{\perp}$. Suppose $V_{z}\left(H_{0}+i\right)^{-1}$ is uniformly bounded on compacts of the strip. Then $V_{z}\left(H_{0}+i\right)^{-1}$ is an analytic operator-valued function of $z$ for $|\operatorname{Im} z|<\beta$.

The proof of Lemma 4.5 is an easy application of the Cauchy integral formula and Fubini's theorem which show that if the circle $\left\{z:\left|z-z_{0}\right| \leqq r\right\}$ is contained in the strip, then

$$
\left(\phi, V_{z}\left(H_{0}+i\right)^{-1} \psi\right)=(2 \pi i)^{-1} \oint_{\left|z^{\prime}-z_{0}\right|=r} d z^{\prime}\left(\phi, V_{z},\left(H_{0}+i\right)^{-1} \psi\right) /\left(z^{\prime}-z\right) .
$$


Definition 4.6. If $A$ is a closed operator, we define $\sigma_{\text {discrete }}(A)$ as the set of all $\lambda_{0}$ such that $\lambda_{0}$ is an isolated point of $\sigma(A)$ and $P_{\lambda_{0}}=-(2 \pi i)^{-1} \oint_{\left|\lambda-\lambda_{0}\right|=\varepsilon}(A-\lambda)^{-1} d \lambda$ is a finite dimensional projection. We define $\sigma_{\text {essential }}(A)=\sigma(A) \backslash \sigma_{\text {discrete }}(A)$

We remark that if $A$ is not self-adjoint, there are definitions of $\sigma_{\text {essential }}(A)$ in the literature which differ from ours [11]. We note without proof that if $A$ is self-adjoint and $B(A+i)^{-1}$ is compact, $\left(B\right.$ need not be symmetric) then $(A+B) \uparrow_{\mathscr{D}(A)}$ is closed and $\sigma_{\text {essential }}(A)=\sigma_{\text {essential }}(A+B)[18]$.

Theorem 4.7. Suppose $V$ is $H_{0}$-translation analytic in the strip $|\operatorname{Imz}|<\beta$. Let $H=$ $-\Delta+\varepsilon_{0} x+V, \varepsilon_{0}>0$. Then

a) The point spectrum of $H$ does not have a finite point of accumulation;

b) $\sigma_{\text {sing. cont. }}(H)=\phi$;

c) $\sigma_{\text {abs.cont. }}(H)=(-\infty, \infty)$.

The family of operators $H_{z}=-\Delta+\varepsilon_{0}(x+z)+V_{z}$ is an analytic family of type $A$ in the sense of Kato [11] and has the following spectral properties

(i) $\sigma_{\text {essential }}\left(H_{z}\right)=R+i \varepsilon_{0}(\operatorname{Imz})$;

(ii) $\sigma_{\text {discrete }}\left(H_{z}\right) \cong\left\{\lambda: \operatorname{Im} \lambda \in\left[0, \varepsilon_{0} \operatorname{Imz}\right)\right\}$;

(iii) The singularities of $\left(H_{z}-\lambda\right)^{-1}$ in the variable $\lambda$ which occur in the region $\operatorname{Im} \lambda \in\left[0, \varepsilon_{0} \operatorname{Imz}\right)$ are poles with finite rank residues whose locations are independent of $z$.

Remarks. a) The poles of $\left(H_{z}-\lambda\right)^{-1}$ off the real axis are candidates for resonances. A similar situation occurs in the Balslev-Combes analysis and there (for a subclass of dilatation analytic potentials) Simon proved [21] that the locations of these poles coincided with those of poles in the scattering amplitude as a function of complex energy. One is hampered in the Stark effect because a useful independent definition of resonance does not seem to exist.

b) The theorem does not cover $V(x)=-Z /|x|$ but does cover for example $V(\boldsymbol{x})=\int(\varrho(\boldsymbol{y}) /|\boldsymbol{x}-\boldsymbol{y}|) d^{3} y$ where $\varrho(\boldsymbol{y})=-Z\left(2 \pi \sigma^{2}\right)^{-3 / 2} \exp \left\{-\frac{1}{2} \boldsymbol{y}^{2} / \sigma^{2}\right\}$ which as $\sigma \rightarrow 0$ approaches $-Z \delta(y)$. It is conceivable that the "resonance" positions converge as $\sigma \rightarrow 0$ to those associated with the Stark effect.

Proof of Theorem 4.7. Because $V_{z}$ is compact relative to $H_{0}$, we have

$$
\sigma_{\text {essential }}\left(H_{z}\right)=\sigma_{\text {essential }}\left(H_{0}+V_{z}\right)+\varepsilon_{0} z=\mathbb{R}+i \varepsilon_{0}(\operatorname{Imz})
$$

if $|\operatorname{Imz}|<\beta$. The discrete spectrum of $H_{z}$ is our next problem. Suppose $\lambda_{0}(z)$ is an eigenvalue of $H_{z}, \lambda_{0} \in \sigma_{\text {discrete }}\left(H_{z}\right)$. Then since $\left\{H_{z}\right\}$ is (by Kato's definition [11]) an analytic family of operators (of type $A$ ) $\lambda_{0}$ is analytic in $z$ (except for certain special values of $z$ in a discrete set). Since $U_{\alpha} H_{z} U_{\alpha}^{-1}=H_{z+\alpha}$, where $\left(U_{\beta} f\right)\left(x, x_{\perp}\right)=$ $f\left(x+\beta, x_{\perp}\right)$ and is thus unitary (for real $\alpha$ ), we have $\lambda_{0}(z+\alpha)=\lambda_{0}(z)$ in a region of analyticity of $\lambda_{0}$ and thus $\lambda_{0}=$ constant. [Note also that $\lambda_{0}(z)$ is a continuous function of $z$ and thus cannot jump at the above-mentioned special values of $z$.] Suppose $\lambda_{0}$ is an eigenvalue of $H_{z}$ and $\lambda_{0}$ is outside the set $\left[0, \varepsilon_{0} \operatorname{Imz}\right]$. Consider the projection

$$
P_{z}=(2 \pi i)^{-1} \oint_{r=\left|\lambda-\lambda_{0}\right|} \frac{d \lambda}{\lambda-H_{z}}
$$


where $r$ is small enough so that no other eigenvalues or essential spectrum is contained within or on the circle $r=\left|\lambda-\lambda_{0}\right|$. Then $P_{z}$ is analytic in $z$ and at $\operatorname{Imz}=0$ must be zero since $H_{z}$ is self-adjoint for $\operatorname{Imz}=0$. But since dimension $\left(P_{z}\right)=$ constant, $P_{z}=0$ and thus $\lambda_{0}$ is not an eigenvalue. This proves (i) through (iii).

Let $\mathscr{D}=\left\{\psi \in L^{2}: U_{\alpha} \psi\right.$ is entire in $\left.\alpha\right\}$, and suppose $\varepsilon>0$. Consider for $\psi \in \mathscr{D}$, the expression (with $\lambda$ real)

$$
\left(\psi,(H-\lambda+i \varepsilon)^{-1} \psi\right)=\left(U_{\alpha} \psi,\left(H_{\alpha}-\lambda+i \varepsilon\right)^{-1} U_{\alpha} \psi\right) .
$$

Equation (4.7) holds for $\alpha$ real but can also be continued to $0<\operatorname{Im} \alpha<\beta$. Thus if the interval $[\mathrm{a}, \mathrm{b}]$ contains no poles of $\left(H_{\alpha}-z\right)^{-1}$ on the real axis (for $\operatorname{Im} \alpha>0$ ) we have

$$
\sup _{\substack{\lambda \in[a, b] \\ 1 \geqq \varepsilon>0}}\left|\left(\psi,(H-\lambda+i \varepsilon)^{-1} \psi\right)\right|<\infty .
$$

By a standard theorem [18], this implies the spectrum of $H$ in $[a, b]$ is purely absolutely continuous. Since the poles of $\left(H_{\alpha}-z\right)^{-1}$ are discrete for $\operatorname{Im} \alpha>0$, a) and b) of the theorem follow. This combined with the fact that $\sigma_{\text {essential }}(H)=$ $(-\infty, \infty)[$ i.e. Eq. (4.6)] proves part c).

\section{Non Existence of Bound States}

In this section we prove that for a large class of potentials, $V$, the Stark Hamiltonian has no eigenvalues. Intuitively, this follows from a tunnelling argument [13]. However, to illustrate the subtlety of the problem we shall first show that an operator, closely related to the hydrogen Stark Hamiltonian, does have eigenvalues.

Let $\mathscr{H}_{m}$ denote the (closed) subspace of square integrable functions with angular momentum $m$ in the direction of the homogeneous field. $\mathscr{H}_{m}$ is an invariant subspace for the hydrogen Stark Hamiltonian $H . H_{m}$ is the restriction $H \uparrow_{\mathscr{H}_{m} \cap D(H)}$. When implemented in parabolic coordinates, $\mathscr{H}_{m}$ and $H_{m}$ are $[6,13]$

$$
\begin{aligned}
\mathscr{H}_{m} & =\left\{\psi(\phi, \xi, \eta)=\left.e^{i m \phi} \psi(\xi, \eta)\left|\int_{0}^{\infty} d \xi \int_{0}^{\infty} d \eta r\right| \psi(\xi, \eta)\right|^{2}<\infty\right\} \\
H_{m} & =\frac{1}{r}\left[-\partial_{\xi} \xi \partial_{\xi}-\partial_{\eta} \eta \partial_{\eta}+\frac{m^{2}}{4}\left(\xi^{-1}+\eta^{-1}\right)-z+\varepsilon_{0}\left(\xi^{2}-\eta^{2}\right)\right] \\
r & =\xi+\eta,
\end{aligned}
$$

$z$ is the charge of the nucleus, $\varepsilon_{0}$ the homogeneous field. This operator was analyzed by Titchmarsh [23] who showed that it has no eigenvalues. However, the differential equation $\left(H_{m}-E\right) \psi=0$ has for some real $E$ non-trivial solutions $\psi$ with a finite tensor product norm:

$$
\|\psi\|^{2}=\int_{0}^{\infty} d \xi \int_{0}^{\infty} d \eta|\psi(\xi, \eta)|^{2}<\infty .
$$

Indeed, the unitary operator $U$ on $L^{2}(0, \infty)$ defined by

$$
U \psi(\xi)=(\xi / 2)^{1 / 2} \psi\left(\xi^{2} / 4\right)
$$


transforms the formal differential operators

$$
\begin{aligned}
& A_{m}=-\partial_{\xi} \xi \partial_{\xi}+m^{2} / 4 \xi+\varepsilon_{0} \xi^{2}-E \xi \\
& B_{m}=-\partial_{\xi} \xi \partial_{\xi}+m^{2} / 4 \xi-\varepsilon_{0} \xi^{2}-E \xi
\end{aligned}
$$

to

$$
\begin{aligned}
& U A_{m} U^{-1}=-\partial_{\xi \xi}+\left(m^{2}-\frac{1}{4}\right) / \xi^{2}+\varepsilon_{0} \xi^{4} / 16-E \xi^{2} / 4 \\
& U B_{m} U^{-1}=-\partial_{\xi \xi}+\left(m^{2}-\frac{1}{4}\right) / \xi^{2}-\varepsilon_{0} \xi^{4} / 16-E \xi^{2} / 4 .
\end{aligned}
$$

Much is known about the spectral properties of differential operators of this type [7]. In particular, when $A_{m}$ and $B_{m}$ are properly defined as self-adjoint operators on the half-line, their essential spectra are empty. For $A_{m}$ this agrees with naive intuition. For $B_{m}$ this is somewhat surprising and is related to the finite travel time to infinity of the corresponding classical particles. It follows that $H_{m}-E$ has non-trivial eigenfunctions in the (linear) tensor product space (at least for some $z$ ).

Non-existence of eigenvalues for the Stark Hamiltonian was first proved by Titchmarsh for the hydrogen Stark effect [23]. His proof is quite involved and relies on the special symmetry (the separability in parabolic coordinates) of the problem. The non-existence of eigenvalues for a wide class of potentials was announced by Agmon [1]. Although he subsequently published similar theorems [2], a proof of the relevant result (Theorem 8 of [1]) has not, to our knowledge, appeared in the literature.

In the spectral theory of Schrödinger operators, two techniques have been developed to prove absence of eigenvalues. One, due to Weidmann and Lavine, was invented to handle (multiparticle) repulsive potentials and is technically based on the existence of an operator which has a positive commutator with $H$ $[14,24]$. The second, due to Kato [12], was introduced to prove absence of positive eigenvalues and is based on the analysis of a rather artificial-looking object $[9,20]$. It so turns out that Kato's ideas can be extended to Stark Hamiltonians to prove absence of eigenvalues for a large class of potentials, $V$. Unfortunately, the proof is involved. On the other hand, techniques of positive commutators handle only special classes of potentials. We shall start by mentioning two results of this type because their extreme formal simplicity contrasts nicely with the general analysis (via Kato's method) to be presented subsequently. [No attempt at rigor will be made in (a) and (b) below.]

(a) Repulsive cores: Let $\varrho=\left|\boldsymbol{x}_{\perp}\right|, \boldsymbol{x}_{\perp} \in \mathbb{R}^{2}$ ( $\boldsymbol{x}_{\perp}$ is in the plane perpendicular to the homogeneous field) and $\partial V / \partial \varrho \leqq 0$ in some suitable (distributional) sense. Let $U_{\alpha}$ be the dilation

$$
U_{\alpha} \psi\left(x, \boldsymbol{x}_{\perp}\right)=\psi\left(x, \alpha \boldsymbol{x}_{\perp}\right) \alpha
$$

and $\psi \in \mathscr{D}\left(\boldsymbol{p}^{2}\right) \cap \mathscr{D}\left(\boldsymbol{p}^{2}+V-\varepsilon_{0} x\right)$ satisfying

$$
H \psi \equiv\left(\boldsymbol{p}^{2}+V-\varepsilon_{0} x\right) \psi=E \psi .
$$

Then $\psi \equiv 0$. In fact

$$
0=\left(U_{\alpha} \psi,\left[U_{\alpha} H U_{\alpha}^{-1}-H\right] \psi\right)
$$


and

$$
\lim _{\alpha \rightarrow 1}(\alpha-1)^{-1}\left(U_{\alpha} \psi,\left[U_{\alpha} H U_{\alpha}^{-1}-H\right] \psi\right)=-\left(\psi,\left[2 p_{\perp}^{2}-\varrho \partial V / \partial \varrho\right] \psi\right)=0
$$

imply $\psi \equiv 0$.

(b) A continuously differentiable function $V(\boldsymbol{x})$ is a classically non-binding potential if $\exists \boldsymbol{n} \in \mathbb{R}^{n}$ such that $\nabla_{\boldsymbol{n}} V(\boldsymbol{x})$ is of fixed sign. Let $U_{\alpha}$ be the unitary translation in the $\boldsymbol{n}$ direction, $V$ classically non-binding, and $\psi \in \mathscr{D}\left(\boldsymbol{p}^{2}\right) \cap \mathscr{D}\left(\boldsymbol{p}^{2}+V\right)$

$$
\left(p^{2}+V\right) \psi=E \psi \text {. }
$$

Mimicking the argument in (a) one finds $\left(\psi, \nabla_{n} V \psi\right)=0$. Hence $\psi=0$ on the support of $\nabla_{n} V$. For $V$ satisfying the hypotheses of a unique continuation theorem, $\psi=0$ (Theorem 5.2).

We shall now turn to the general analysis. In the following it is convenient to choose the field in the positive $x$ direction, i.e.

$$
H_{0}=-\Delta-x \quad\left(\boldsymbol{x}=\left(x, \boldsymbol{x}_{\perp}\right)\right) .
$$

$H=H_{0}+V$, with $V$ satisfying the following regularity conditions:

A. $V=V_{1}+V_{2}, V_{1} \in L^{2}\left(\mathbb{R}^{3}\right), V_{1}(\boldsymbol{x})=0$ if $x \geqq R$ $V_{2} \in L^{\infty}\left(\mathbb{R}^{3}\right)$, for some $R>0$.

B. There is an $x_{0}>0$ so that for $x \geqq x_{0}$ the operator $V_{x}$ defined on $L^{2}\left(\mathbb{R}^{2}\right)$ by

$$
V_{x} \psi\left(\boldsymbol{x}_{\perp}\right)=V(\boldsymbol{x}) \psi\left(\boldsymbol{x}_{\perp}\right)
$$

is bounded and strongly differentiable in $x$ with a derivative $V_{x}^{\prime}$ satisfying

$$
\left\|V_{x}^{\prime}\right\| \leqq c, \quad \text { if } \quad x \geqq x_{0} .
$$

There are two distinct results which taken together guarantee the absence of eigenvalues. The first says that a particle cannot remain indefinitely in regions of space where there is a net force pushing it out to infinity. This is the subject of the following theorem:

Theorem 5.1. Suppose $V$ satisfies $A, B$ and in addition for $x \geqq x_{0}, V_{x}^{\prime} \leqq 1-\delta$, for some $\delta>0$. If $(H-E) \psi=0$ and $\psi \in \mathscr{D}(H)$ then $\psi(x)=0$ for all $x \geqq x_{0}$.

The second result says that for certain $V$ 's a quantum mechanical particle will always "tunnel" through classically forbidden regions. (It will thus "leak out" to regions of space when the force accelerates it to infinity.) This is a well known result from the theory of partial differential equations [3]. Unfortunately the requirements on $V$ are rather strong (perhaps unrealistically so).

Theorem 5.2. Suppose $S$ is a closed set of measure zero with $\mathbb{R}^{3} \backslash S$ connected. Suppose $W$ is a function bounded on compacts of $\mathbb{R}^{3} \backslash S$. If $\psi \in L^{2}\left(\mathbb{R}^{3}\right) \cap\{\phi: f \phi \in$ $\mathscr{D}(-\Delta)$ for each $\left.f \in C_{0}^{\infty}\left(\mathbb{R}^{2}\right)\right\}$ and $\Delta \psi=W \psi$ as distributions, then if $\psi$ vanishes on an open subset of $\mathbb{R}^{3}, \psi \equiv 0$. 
As a corollary we thus have

Theorem 5.3. Suppose $V$ satisfies the assumptions of Theorem 5.1 and in addition is bounded on compacts of $\mathbb{R}^{3} \backslash S, S$ a closed set of measure zero which does not disconnect $\mathbb{R}^{3}$. Then $H=H_{0}+V$ has no eigenvalues.

Before proceeding with a proof of Theorem 5.1 we need a technical result:

Proposition 5.4. Suppose $V$ satisfies $A, B$. Let $H=H_{0}+V$.

a) If $f: \mathbb{R}^{3} \rightarrow \mathbb{C}$ and $\left(x^{2}+1\right)^{1 / 2}\left(|f|+\sum_{j}\left|\partial_{j} f\right|\right)+|\Delta f|$ is bounded then $\left\|\left(\boldsymbol{p}^{2}+1\right) f(H+i)^{-1}\right\|<\infty$.

b) If $g \in C_{0}^{\infty}\left(\mathbb{R}^{1}\right)$ with $\operatorname{supp} g \subseteq\left\{x: x \geqq x_{0}\right\}$ then

$\left\|p_{1}\left(\boldsymbol{p}^{2}+1\right) g(H+i)^{-2}\right\|<\infty$.

A proof of Proposition 5.4 is sketched in Appendix A.

Proof of Theorem 5.1. Suppose $H \psi=E \psi$. Without loss of generality we can assume that $\psi$ is real-valued. The restriction of $\psi, \psi_{x}$, to a fixed value of $x$ has certain regularity properties by virtue of Proposition 5.4:

(i) $\psi_{x}$ is a twice continuously differentiable $L^{2}\left(\mathbb{R}^{2}\right)$ valued function of $x$.

(ii) Let $p_{j}=-i \nabla_{j}$ and $\boldsymbol{p}_{\perp}^{2}=p_{2}^{2}+p_{3}^{2}$. Then $\psi_{x} \in \mathscr{D}\left(\boldsymbol{p}_{\perp}^{2}\right)$ and $p_{j} \psi_{x}$ is a continuously differentiable $\left[L^{2}\left(\mathbb{R}^{2}\right)\right.$ valued] function of $x$.

(iii)

$$
\int_{x_{0}}^{\infty} x^{-1} d x\left(\sum_{j=2}^{3}\left\|p_{j} \psi_{x}\right\|^{2}+\left\|\psi_{x}^{\prime}\right\|^{2}\right)<\infty .
$$

Here the prime indicates differentiation with respect to $x$. Statements (i) and (ii) follow from the easily proved inequalities.

$$
\begin{aligned}
\left\|\phi_{x}\right\| & \leqq c_{\alpha}\left\|\left(p_{1}^{2}+1\right)^{\alpha} \phi\right\|, \alpha>1 / 4 \\
\left\|\phi_{x}-\phi_{y}\right\| & \leqq c_{\alpha}\left\|\left(p_{1}^{2}+1\right)^{\alpha}\left(e^{i p_{1}(x-y)}-1\right) \phi\right\| \\
& \leqq c_{\alpha \beta}\left\|\left(p_{1}^{2}+1\right)^{\alpha+\beta} \phi\right\||x-y|^{2 \beta}, \alpha>1 / 4, \beta<1 / 2
\end{aligned}
$$

and from part (b) of the proposition. Statement (iii) follows from the fact that

$$
\left\|p_{j}\left(x^{2}+1\right)^{-1 / 2} p_{\ell}(H+i)^{-1}\right\|<\infty
$$

which in turn follows from (a) of the proposition. These results are needed to justify the manipulations to be carried out below.

The central idea in the positive commutator technique for proving absence of eigenvalues is the search for a dynamical variable that increases in time. Our approach is, in some sense, a special, local version of this idea. One starts from the observation that for $x \geqq x_{0}$, the expectation value, in the plane perpendicular to the field, of the force in the $x$-direction is non-negative. Let $f:\left[x_{0}, \infty\right) \rightarrow \mathbb{R}$ be given by

$$
f(x) \equiv\left\langle\psi_{x},\left(1-V_{x}^{\prime}\right) \psi_{x}\right\rangle \geqq \delta\left\langle\psi_{x}, \psi_{x}\right\rangle \geqq 0
$$

where

$$
\psi_{x}^{\prime \prime}=\boldsymbol{p}_{\perp}^{2} \psi_{x}+\left(V_{x}-x-E\right) \psi_{x} .
$$


$f$ is integrable on $\left[x_{0}, \infty\right)$, hence we consider the expectation of the force acting on the "part of the particle" to the right of $x$ :

$$
\int_{x}^{\infty} f(y) d y=G(x)+c_{0}
$$

where

$$
G(x) \equiv-\left\|\psi_{x}^{\prime}\right\|^{2}+\left\langle\psi_{x},\left(\boldsymbol{p}_{\perp}^{2}+V_{x}-x-E\right) \psi_{x}\right\rangle .
$$

Equation (5.4) follows from the Schrödinger Equation (5.3). A function similar to $G$ was originally introduced by Kato [12]. We will henceforth analyze the quantity $G$ and other similar quantities (it will shortly be shown that the quantity $c_{0}$ in (5.4) is zero). Our procedure will be to show that if $\gamma^{3}<2 \delta$ then

$$
\infty>\int_{x_{0}}^{\infty} d x e^{\gamma x}\left\{\left\|\psi_{x}^{\prime}\right\|^{2}+\left\langle\psi_{x}, \boldsymbol{p}_{\perp}^{2} \psi_{x}\right\rangle+x\left\langle\psi_{x}, \psi_{x}\right\rangle\right\} .
$$

This will lead to a contradiction unless $\psi_{x}=0$ for $x>x_{0}$.

First note that if $G$ is negative at some $x_{1} \geqq x_{0}$, then by (5.4) and (5.2) $|G(x)| \geqq$ $\left|G\left(x_{1}\right)\right|>0$ for all $x \geqq x_{1}$. If $G$ is positive for all $x \geqq x_{0}$ but $\lim _{x \rightarrow \infty} G(x)>0$, then $|G(x)| \geqq$ $\lim _{y \rightarrow \infty} G(y)>0$ all $x \geqq x_{0}$. In either case (5.1) implies

$$
\begin{aligned}
\infty=\int_{x_{0}}^{\infty}|G(x)| x^{-1} d x & \leqq \int_{x_{0}}^{\infty} x^{-1} d x\left\{\left\|\psi_{x}^{\prime}\right\|^{2}+\left\langle\psi_{x}, \boldsymbol{p}_{\perp}^{2} \psi_{x}\right\rangle\right. \\
& \left.+\left\langle\psi_{x},\left(\left|V_{x}\right|+x+|E|\right) \psi_{x}\right\rangle\right\}<\infty,
\end{aligned}
$$

a contradiction. We have thus shown

$$
G(x) \geqq 0, \quad \lim _{x \rightarrow \infty} G(x)=0 .
$$

(This means that $c_{0}=0$.) Introducing the function $g(x)=\left\langle\psi_{x}, \psi_{x}\right\rangle,(5.2)$ then gives

$$
G(x) \geqq \delta \int_{x}^{\infty} d y g(y) .
$$

Note also that

$$
g^{\prime \prime}(x)=4\left\|\psi_{x}^{\prime}\right\|^{2}+2 G(x) \geqq 0 .
$$

Since $g$ is a non-negative integrable function it is easy to see that $g^{\prime}(x) \leqq 0, \lim _{x \rightarrow \infty} g(x)=$ $\lim _{x \rightarrow \infty} g^{\prime}(x)=0$.

$$
g(x)=\int_{x}^{\infty} d y_{1} \int_{y_{1}}^{\infty} d y_{2} g^{\prime \prime}\left(y_{2}\right)
$$

and by Fubini's theorem (5.10) implies

$$
\int_{x}^{\infty} g(y) d y=\int_{x}^{\infty} d y(y-x)^{2}\left(2\left\|\psi_{y}^{\prime}\right\|^{2}+G(y)\right) .
$$


Equation (5.8) then gives

$$
G(x) \geqq \delta \int_{x}^{\infty} d y(y-x)^{2}\left[2\left\|\psi_{y}^{\prime}\right\|^{2}+G(y)\right] .
$$

Using $g^{\prime \prime}(x) \geqq 2 G(x) \geqq 2 \delta \int_{x}^{\infty} d y g(y)$ and Equation (5.10) results in the inequality

$$
g(x) \geqq \delta \int_{x}^{\infty} d y(y-x)^{2} g(y)
$$

Note that from Equation (5.12), $G$ also satisfies this inequality. We next show that any non-negative (finite valued) function, $h$, satisfying (5.13) also satisfies

$$
\int_{x_{0}}^{\infty} d x e^{\gamma x} h(x)<\infty \quad \gamma^{3}<2 \delta .
$$

We first multiply both sides of Equation (5.13) by $(y-x)^{n}$ and integrate to find

$$
c_{n} \equiv \int_{x_{0}}^{\infty} d y(n !)^{-1}\left(y-x_{0}\right)^{n} h(y) \geqq 2 \delta \int_{x_{0}}^{\infty} d y((n+3) !)^{-1}\left(y-x_{0}\right)^{n+3} h(y)=2 \delta c_{n+3}
$$

where $c_{n}$ is finite for all $n$ by induction. Equation (5.15) gives $c_{3 n+j} \leqq(2 \delta)^{-n} c_{j}$, $j=0,1,2$ so that for $\gamma^{3}<2 \delta$

$$
\int_{x_{0}}^{\infty} d y e^{\gamma\left(y-x_{0}\right)} h(y)=\sum_{n=0}^{\infty} \gamma^{n} c_{n} \leqq\left(\sum_{n=0}^{2} c_{n} \gamma^{n}\right)\left(1-\gamma^{3} / 2 \delta\right)^{-1} .
$$

This proves Equation (5.14). Also note that from Equation (5.12)

$$
\int_{x_{0}}^{\infty} e^{\gamma x}\left\|\psi_{x}^{\prime}\right\|^{2} d x<\infty \quad \gamma^{3}<2 \delta
$$

Thus we have proved Equation (5.6). We complete the proof by showing that Equation (5.6) is impossible unless $\psi_{x}=0$ for $x>x_{0}$ :

Let $\phi_{x} \equiv \phi_{x, \varepsilon} \equiv \psi_{x} \exp \left(\lambda x^{1-\varepsilon}\right)$ and note that $\phi_{x}$ satisfies the equation

$$
\phi_{x}^{\prime \prime}=2 \phi_{x}^{\prime} \lambda(1-\varepsilon) x^{-\varepsilon}+\left(p_{\perp}^{2}+V_{x}-(x+E)-W_{x}\right) \phi
$$

where

$$
W_{x} \equiv W_{x, \lambda, \varepsilon} \equiv \lambda \varepsilon(1-\varepsilon) x^{-1-\varepsilon}+\lambda^{2}(1-\varepsilon)^{2} x^{-2 \varepsilon}
$$

We next introduce the function

$$
G_{\lambda, \varepsilon}(x) \equiv-\left\|\phi_{x}^{\prime}\right\|^{2}+\left\langle\phi_{x},\left(p_{\perp}^{2}+V_{x}-(x+E)-W_{x}\right) \phi_{x}\right\rangle
$$

and compute

$$
G_{\lambda, \varepsilon}^{\prime}(x)=-4 \lambda(1-\varepsilon) x^{-\varepsilon}\left\|\phi_{x}^{\prime}\right\|^{2}+\left\langle\phi_{x},\left(-1+V_{x}^{\prime}-W_{x}^{\prime}\right) \phi_{x}\right\rangle
$$

where

$$
-W_{x}^{\prime}=\lambda \varepsilon\left(1-\varepsilon^{2}\right) x^{-2-\varepsilon}+2 \varepsilon \lambda^{2}(1-\varepsilon)^{2} x^{-1-2 \varepsilon} .
$$


Suppose $\psi_{x_{1}} \neq 0$, for some $x_{1} \geqq x_{0}$. Choose $\lambda_{0}$ so large that $G_{\lambda_{0}, \varepsilon}\left(x_{1}\right)<0$ for all $\varepsilon$ in the interval $(0,1 / 2)$. Choose $\varepsilon_{0}>0$ so small that

$$
-W_{x_{1}, \lambda_{0}, \varepsilon_{0}}^{\prime}<\delta / 2 \text {. }
$$

Then since $-W_{x}^{\prime}$ is a decreasing function of $x$

$$
G_{\lambda_{0}, \varepsilon_{0}}^{\prime}(x) \leqq-(\delta / 2)\left\langle\phi_{x}, \phi_{x}\right\rangle \leqq 0 \quad \text { all } \quad x \geqq x_{1} .
$$

Thus $G_{\lambda_{0}, \varepsilon_{0}}(x)<G_{\lambda_{0}, \varepsilon_{0}}\left(x_{1}\right)<0$ all $x>x_{1}$. In particular $G_{\lambda_{0}, \varepsilon_{0}}(x)$ is not integrable on $\left[x_{0}, \infty\right)$ in contradiction to Equation (5.6). This proves Theorem 5.1.

Acknowledgement. A preliminary version of this work was presented at a colloquium by one of us (I.W.H.) at the Physics Department of the University of New Mexico. The interest and useful comments of the audience are gratefully acknowledged.

\section{Appendix. Operator Bounds}

We first consider the operator $\left(\boldsymbol{p}^{2}+1\right) f(H+i)^{-1}$. Using $\left[\boldsymbol{p}^{2}, f\right]=\sum_{j} 2 p_{j} f_{j}+\Delta f$, $f_{j}=-i \partial_{j} f$ it is easy to derive

$$
\begin{aligned}
\left(\boldsymbol{p}^{2}+1\right) f(H+i)^{-1}= & \sum_{j} 2 p_{j} f_{j}(H+i)^{-1}+(f(1-i+x)+\Delta f-f V) \\
& \cdot(H+i)^{-1}+f .
\end{aligned}
$$

On the other hand

$$
\begin{aligned}
p_{j} g\left(H_{0}+i\right)^{-1}= & g p_{j}\left(p^{2}+i\right)^{-1}+x g p_{j}\left(p^{2}+i\right)^{-1}\left(H_{0}+i\right)^{-1} \\
& +g\left[p_{j}\left(p^{2}+i\right)^{-1}, x\right]\left(H_{0}+i\right)^{-1}+g_{j}\left(H_{0}+i\right)^{-1} .
\end{aligned}
$$

We also have

$$
\left\|\left(H_{0}+i\right)(H+i)^{-1}\right\|<\infty
$$

so that the first term of (A1) is bounded because of (A2), while the second term is bounded because $\left\|V(H+i)^{-1}\right\|<\infty$.

To prove part (b) it is enough by (A3) to show that

$$
\left\|p_{1}\left(\boldsymbol{p}^{2}+1\right) f(H+i)^{-1}\left(H_{0}+i\right)^{-1}\right\|<\infty \text {. }
$$

We use (A1) and note that

$$
p_{1} p_{j} f_{j}(H+i)^{-1}
$$

is bounded by part (a) of the proposition. The expressions

$$
p_{1}(f(1+i+x)+\Delta f+f V)(H+i)^{-1}
$$

and

$$
p_{1} f\left(H_{0}+i\right)^{-1}
$$

are bounded by (A2). 


\section{References}

1. Agmon, S.: Proceedings of the Tokyo Int. Conf. on Functional Analysis and Related Topics, 1969

2. Agmon, S.: J. d'Analyse, Math. 23, 1-25 (1970)

3. Aronszajn, N.: J. Math. Pures Appl. 36, 235-249 (1957)

4. Balslev,E., Combes, J. M.: Commun. math. Phys. 22, 280-294 (1971)

5. Chandler, C., Stapp, H.: J. Math. Phys. 10, 826-859 (1969)

6. Condon,E.U., Shortley, G.H.: The theory of atomic spectra. Cambridge: Cambridge University Press 1970

7. Dunford, N., Schwartz, J.T.: Linear operators, Vol. II. New York: Interscience 1958

8. Herbst, I.: Commun. math. Phys. 35, 193-214 (1974)

9. Jansen, K. H., Kalf, H.: Comm. Pure Appl. Math. 28, 747-752 (1975)

10. Kato, T.: Trans. Am. Math. Soc. 70, 195-211 (1951)

11. Kato, T.: Perturbation theory for linear operators. Berlin-Heidelberg-New York: Springer 1966

12. Kato, T.: Comm. Pure Appl. Math. 12, 403-425 (1959)

13. Landau, L.D., Lifshitz,E.M.: Quantum mechanics. Oxford: Pergamon 1954

14. Lavine, R.: Commun. math. Phys. 20, 301-323 (1971)

15. Lieb,E.H., Simon, B., Wightman, A.S.: Studies in mathematical physics, essays in honor of V. Bargmann. Princeton: Princeton University Press 1976

16. Rauch, J., Reed, M.: Commun. math. Phys. 29, 105-111 (1973)

17. Reed, M., Simon, B.: Methods of modern mathematical physics, Vol. I, II, New York-London: Academic Press 1974, 1975

18. Reed, M., Simon, B.: Methods of modern mathematical physics, Vol. III, to appear

19. Ruelle, D.: Helv. Phys. Acta 35, 147 (1962)

20. Simon, B.: Comm. Pure. Appl. Math. 22, 531-538 (1969)

21. Simon, B.: Ann. Math. 97, 247-274 (1973)

22. Simon, B. : Commun. math. Phys. 23, 37 (1971)

23. Titchmarsh,E.C.: Eigenfunction expansions associated with second order differential equations. Oxford: Oxford University Press 1958

24. Weidmann, J.: Bull. AMS 73, 452-456 (1967)

25. Wilcox,C.H.: Perturbation Theory and Its Application in Quantum Mechanics. New York-London: J. Wiley 1966

Communicated by W. Hunziker

Received September 3, 1976

\section{Note Added in Proof}

We would also like to mention the recent results of P. Rejto and K. Sinha (Helv. Phys. Acta 49, 389-413 (1976)) who prove the absolute continuity of the spectrum of a class of one-dimensional Stark-like Hamiltonians. 